\title{
Estudo preditibo da intensidade de corrida na meia-maratona com base na relação lactato- velocidade obtida num teste de terreno
}

\author{
Paulo J. M. Santos*
}

\begin{abstract}
Resumo
O objetivo deste estudo foi investigar a validade de um teste de terreno na predição da performance na meia-maratona (21095m). Foram avaliados 18 fundistas que realizaram um total de 33 meias-maratonas e um número idêntico de testes de terreno incrementais $(4 \times 2000 \mathrm{~m})$, de modo a determinar a relação entre a intensidade de corrida e a acumulação sangüínea de lactato. As velocidades utilizadas no teste variaram entre 4.2 e $5.8 \mathrm{~m} / \mathrm{s}$, com incrementos de $0.4 \mathrm{~m} / \mathrm{s}$ por patamar. Após cada patamar de carga foram recolhidas amostras de sangue capilar do lóbulo da orelha e analisadas num YSI-1500L Sport para determinação da lactatemia. $\mathrm{Na}$ análise estatística recorremos ao Coeficiente de Correlação de Pearson e ao Modelo de Regressão Linear Simples. O nível de significância foi mantido em $5 \%$. O tempo médio dispendido pelos corredores da nossa amostra na meia-maratona foi de $1 \mathrm{~h} 05^{\prime} 41^{\prime \prime} \pm 307 "$. As velocidades de corrida correspondentes a concentrações de lactato entre 3.0 e $5.5 . \mathrm{mmol} / \mathrm{l}$ evidenciaram uma elevada correlação $(0.86<r<0.90)$ com a velocidade na meia-maratona No entanto, a correlação mais elevada foi encontrada para níveis de lactato de 4.5, 5.0 e 5.5mmol/I $\left(V_{4.5}, V_{5.0}\right.$ e $\left.V_{5.5}\right)$. Com efeito, estes índices parecem poder assegurar as melhores previsões uma vez que o $r^{2}$ encontrado foi de $80 \%$ para a $V_{4.5}$ e de $81 \%$ para as $V_{5.0}-V_{5.5}$, com o mesmo erro padrão de estimativa $(0.1$ 1). No entanto, em termos práticos e apesar da elevada correlação encontrada, os nossos resultados mostram uma eficácia preditiva limitada e uma reduzida aplicabilidade prática. De facto, as previsões baseadas na $V_{3.0}(5.47 \mathrm{~m} / \mathrm{s})$ até à $V_{5.5}(5.78 \mathrm{~m} / \mathrm{s})$ incluem uma margem de erro de $\pm 3^{\prime} 16^{\prime \prime}$ para os
\end{abstract}

melhores corredores. Apenas reduzindo o intervalo de previsão, ou seja, considerando apenas as estimativas baseadas na $V_{4.5^{-}}$ $V_{5.5}$ é que é se torna possível encontrar uma margem de erro aceitável ( \pm 1 '23"). No entanto, é forçoso referir que a análise dos nossos dados revelou que $70 \%$ dos atletas testados apresentou um tempo final de competição fora do intervalo de uma predição baseada na $\mathrm{V}_{4.5}-\mathrm{V}_{5.5}$

\section{Abstract}

The purpose of this study was to investigate the validity of a field test in predicting the half-marathon $(21095 \mathrm{~m})$ performance. Eighteen long-distance runners performed a total of 33 halfmarathons and an equal amount of incrementai field tests $(4 \times 2000 m)$ to established the relationship between running speed and blood lactate leveis. The speeds used in the field test ranged from 4.2 to $5.8 \mathrm{~m} / \mathrm{s}$ with increments of $0.4 \mathrm{~m} / \mathrm{s}$ in each step. Following each loading levei blood samples were taken from the ear lobe and analysed using an YSI-1500L Sport. Statistical analysis included the Pearson correlation coefficient and the simple linear regression model. The levei of significance was set at $5 \%$.

The average time achieved by the runners in the half-marathon was $1 \mathrm{~h}^{\prime} 05^{\prime} 41^{\prime \prime} \pm 3^{\prime} 07^{\prime \prime}$. The test speeds corresponding to a lactate concentration between 3.0 and $5.5 \mathrm{mmol} / \mathrm{l}$ showed an high correlation $(0.86<r<0.90)$ with the half-marathon speed. However the higher correlation $(r=0.90)$ was found at lactate leveis of 4.5 , 5.0 and $5.5 \mathrm{mmol} / \mathrm{l}\left(\mathrm{V}_{4.5}, \mathrm{~V}_{5.0}\right.$ and $\left.\mathrm{V}_{5.5}\right)$. In fact, these values seem to be able to assure the higher predictions since the $r^{2}$ found was of $80 \%$ for 
$\mathrm{V}_{4.5}$ and $81 \%$ for $\mathrm{V}_{50}-\mathrm{V}_{5.5}$, with the same

standard error of estimate $(0.11)$. Nevertheless and in spite of the high correlation found, in practical terms our results showed a limited predictive efficacy and practical applicability. In fact, predictions based on $\mathrm{V}_{3.0}(5.47 \mathrm{~m} / \mathrm{s})$ to $V_{5.5}(5.78 \mathrm{~m} / \mathrm{s})$ include a margin of error of $\pm 3^{\prime} 16 "$ for the top athletes, although estimates based on $V_{4.5}-V_{5.5}$ presented a lower error ( \pm 1 '23"). Additionally $70 \%$ of the studied athletes presented a final competition time outside the interval of the prediction based on the $\mathrm{V}_{4.5}-\mathrm{V}_{5.5}$

\section{Existe uma enorme dificuldade na transferência dos dados do laboratório para o terreno, razão pela qual a investiga- ção nesta área nos últimos anos tem privilegiado, de forma crescente, o estudo do atleta no seu habitat.}

\section{INTRODUÇÃO}

Nas duas últimas décadas vários têm sido os investigadores que têm procurado prever a performance nas corridas de meio-fundo e fundo a partir de um conjunto de indicadores fisiológicos $1,2,3,4,5,7,17,18,19,21,22,23$. No entanto a grande maioria destes pesquisas têm privilegiado a investigação laboratorial em detrimento da avaliação no terreno. Embora o trabalho no laboratório possa, eventualmente, ser mais rigoroso, particularmente por permitir um controle mais eficaz de determinadas variáveis (vento, temperatura, humidade, etc.) e das cargas utilizadas, os dados aí obtidos dificilmente poderão ser utilizados no trabalho de campo do atleta. Com efeito, existe uma enorme dificuldade na transferência dos dados do laboratório para o terreno, razão pela qual a investigação nesta área nos últimos anos tem privilegiado, de forma crescente, o estudo do atleta no seu habitat. Com efeito foram desenvolvidos e validados testes de terreno susceptíveis de serem utilizados no diagnóstico da performance e, simultaneamente, no controle do treino, sem necessidade de recorrer a grande quantidade de equipamento $^{3,4,5,8,9,10,12,14,15,16}$. Entre as principais causas responsáveis por esta mudança de atitude salientaríamos, para além da já referida dificuldade na transferência de dados, os vários tipos de constrangimento que a realização de testes laboratoriais implica para os testados, o que contribui, em grande medida, para a descrença e desmotivação progressiva que habitualmente se observa por parte de atletas e treinadores.
Assim, foi nosso objectivo utilizar um teste de terreno simples mas que, simultaneamente, fornecesse uma grande riqueza de informação susceptível de ser utilizada não apenas no diagnóstico da performance, que é o objetivo fundamental desta investigação, mas igualmente numa perspectiva de avaliação-prescrição, fornecendo ao treinador um instrumento válido de avaliação da intensidade das cargas que o ajude a definir um sistema ou estratégia de treino que lhe permita atingir, de um modo mais rigoroso, os seus objetivos tanto para o treino como para a competição.

Com este objetivo e aproveitando o fato de em Portugal se realizarem anualmente um grande número de meias-maratonas, procuramos determinar se seria possível predizer a performance nesta competição a partir da realização de um único teste de terreno ${ }^{11,20}$. Este teste específico utiliza a relação entre as concentrações sangüíneas de lactato e a velocidade de corrida como critério para determinar as alterações da capacidade aeróbia ${ }^{10}$. Investigações com objetivos semelhantes foram anteriormente conduzidas, mas para tentar predizer a performance na maratona ${ }^{5,17}$.

\section{MATERIAL E MÉTODOS}

Foram avaliados 18 corredores de elite de meio-fundo e fundo com uma média de $24 \pm 3.5$ anos de idade, $174 \pm 5.8 \mathrm{~cm}$ de altura e $60 \pm 7 \mathrm{Kg}$ de peso. A amostra incluía alguns dos melhores especialistas nacionais neste tipo de competições, o que aliás está bem patente nos resultados que obtivemos durante esta pesquisa: média de lh05'41" $\pm 3^{\prime} 07^{\prime \prime}$ (entre lh01'51" elhl2'll").

Utilizamos um teste de terreno incrementai $^{5,20}$, freqüentemente utilizado na determinação do Limiar Aeróbio-Anaeróbio ${ }^{10,}$ u, para determinar a relação entre os vários índices de lactato $(3.0,3.5,4.0,4.5,5.0$ e $5.5 \mathrm{mmol} /$ 1) e a velocidade de corrida $(\mathrm{m} / \mathrm{s})$. Cada teste foi precedido por um aquecimento de 8-10 min de corrida contínua efetuada a baixa velocidade (inferior a $3.0 \mathrm{~m} / \mathrm{s}$ ) para estabilizar as con- 
centrações sangüíneas de lactato. As avaliações foram realizadas numa pista de piso sintético com $400 \mathrm{~m}$, utilizando como percursos $4 \mathrm{x}$ $2.000 \mathrm{~m}$ e incrementos de $0.4 \mathrm{~m} / \mathrm{s}$ por patamar. As velocidades utilizadas foram de 4.2,4.6,5.0, 5.4 e $5.8 \mathrm{~m} / \mathrm{s}$, respectivamente (figura 1 ).

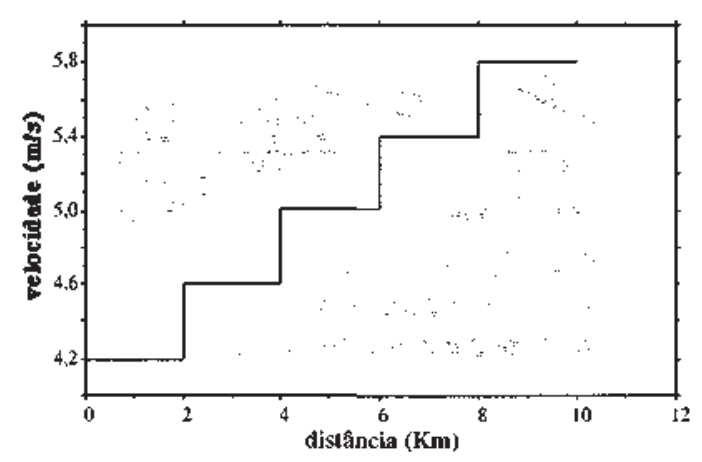

Figura 1 - Representação gráfica da dinâmica de carga referente ao teste de terreno utilizado na avaliação da capacidade aeróbia dos corredores de meio-fundo e fundo. Foram utilizadas distâncias fixas de 2. OOOm por patamar e incrementos de $0,4 \mathrm{~m} / \mathrm{s}$.

Durante a realização de cada patamar eram fornecidos sinais auditivos aos atletas, coincidentes com os tempos de passagem em cada $200 \mathrm{~m}$, para procurar garantir um ritmo de corrida uniforme de modo a evitar flutuações de velocidade. Apesar disso, o tempo final era registado após cada patamar e a velocidade exata calculada. No final de cada $2.000 \mathrm{~m}$ eram efetuadas recolhas de sangue capilar do lóbulo da orelha (30-40s após) e posteriormente analisadas para determinação da concentração sangüínea de lactato num Yellow Springs Instruments 1.500L-Sport. O cálculo da concentração de lactato correspondente a determinada velocidade de corrida contínua foi efetuado por análise da regressão linear a partir dos dados obtidos no teste de terreno $0^{3,10}$.

Determinamos ainda a lactatemia máxima pós-competição, com recolhas sangüíneas efetuadas ao $1^{\circ}, 3^{\circ}, 5^{\circ}, 7^{\circ}, 10^{\circ}$ e $12^{\circ}$ minutos do período de recuperação.

Os corredores da amostra $(n=18)$ efetuaram um total de 33 meias-maratonas e foram igualmente submetidos a 33 testes de ter- reno. Cada teste foi sempre realizado no período máximo de uma semana relativamente à realização da competição. No entanto, a grande maioria teve lugar na semana anterior à competição e somente nos casos em que tal não foi possível é que optamos por efectuar a avaliação na semana imediatamente após a prova. A idéia básica era estabelecermos com grande rigor a relação entre os vários índices de lactato e a performance, motivo pelo qual as avaliações deviam distar temporalmente o menos possível. Gostaríamos ainda de referir que tivemos o cuidado de selecionar provas pouco acidentadas (planas) e bem aferidas em termos de distância.

No tratamento estatístico dos dados recorreu-se ao coeficiente de correlação de Pearson e ao modelo de regressão linear simples. O nível de significância foi mantido em $5 \%$.

\section{RESULTADOS}

Após a realização dos testes de terreno, as velocidades de corrida correspondentes a concentrações fixas de lactato de 3.0, 3.5,4.0, 4.5, 5.0 e $5.5 \mathrm{mmoVl}(\mathrm{V} 3.0, \mathrm{~V} 3.5, \mathrm{~V} 4.0, \mathrm{~V} 4.5, \mathrm{~V} 5.0$ e V5.5) foram correlacionadas com a velocidade a que cada atleta correu a meia-maratona.

O estudo das retas de regressão para as diferentes concentrações de lactato encontrase representado no quadro 1 e na figura 2. A correlação mais elevada $(r=0.90)$ entre o teste de terreno e a velocidade da meia-maratona foi encontrada para as lactatemias de 4.5, 5.0 e $5.5 \mathrm{mmol} / 1$.

Quadro 1 - Estatísticas da relação entre a velocidade de corrida determinada no teste de terreno para concentrações de 3.0, 3.5, 4.0, 4.5, 5.0 e 5.5mmol/l de lactato e a velocidade correspondente à meia-maratona. No quadro encontram-se assinalados os valores de $r, r^{2}$, a equação de regressão e o erro padrão de estimativa.

\begin{tabular}{l|l|l|l|l|}
\hline $\mathbf{V}$ & $\mathrm{r}$ & $\mathrm{r} 2$ & eq. regressão & epe \\
\hline 3.0 & 0.86 & 0.73 & $\mathrm{y}=0.91 \mathrm{x}+0,64$ & 0.13 \\
\hline 3.5 & 0.88 & 0.77 & $\mathrm{y}=0.97 \mathrm{x}+0.26$ & 0.12 \\
\hline 4.0 & 0.89 & 0.79 & $\mathrm{y}=1.00 \mathrm{x}+0.05$ & 0.11 \\
4.5 & 0.90 & 0.80 & $\mathrm{y}=1.00 \mathrm{x}-0.02$ & 0.11 \\
5.0 & 0.90 & 0.81 & $\mathrm{y}=1.01 \mathrm{~T}-0.11$ & 0.11 \\
5.5 & 0.90 & 0.81 & $\mathrm{y}=1.00 \mathrm{x}-0.12$ & 0.11 \\
\hline
\end{tabular}

\section{Os corredores da amostra $(n=18)$ efetuaram um total de 33 meias-marato- nas e foram igual- mente submetidos a 33 testes de terreno.}



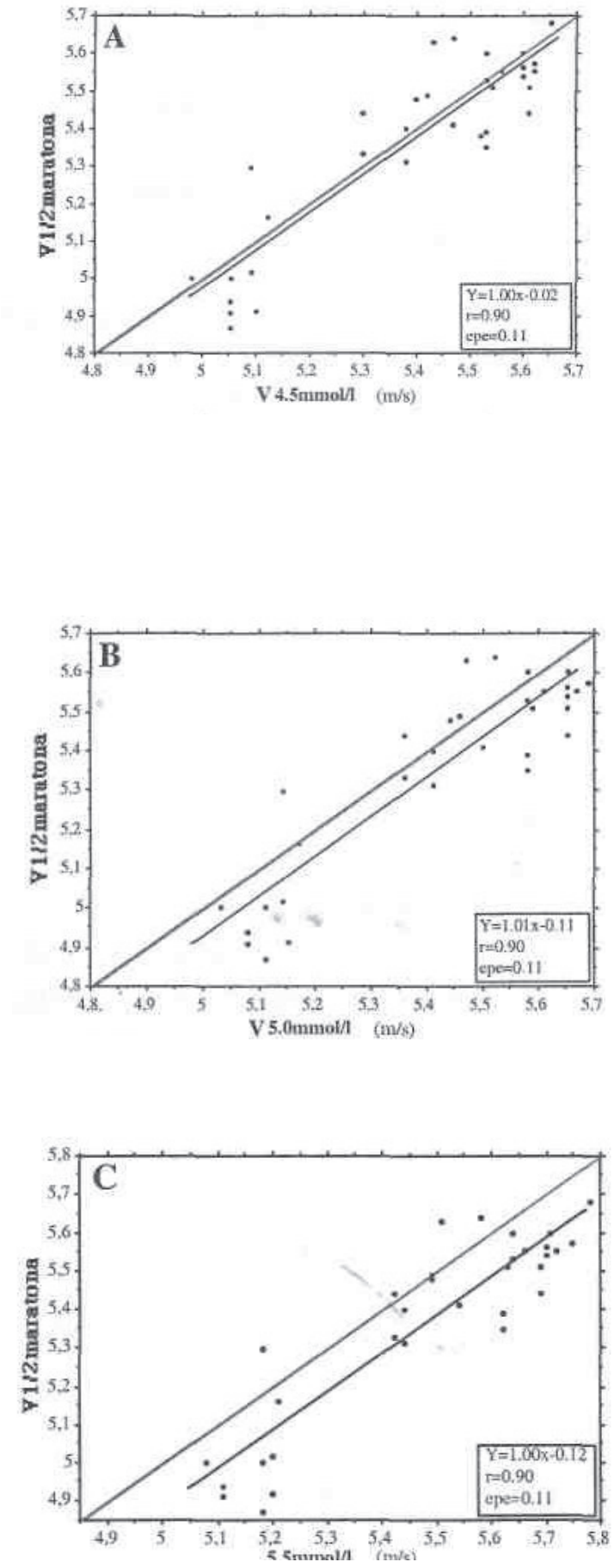

Quadro 2 - Valores médios (土desvio padrão) correspondentes à foram de 80\% (V4.5) e 81\% (V5.0 e velocidade $(\mathrm{m} / \mathrm{s}$ ) na meia-maratona (V1/2mar) e às velocidades $\mathrm{V} 5.5)$, com um mesmo erro padrão de $(\mathrm{m} / \mathrm{s})$ obtidas no teste de terreno para lactatemias situadas entre estimativa de 0.11 (quadro 1).

\begin{tabular}{|l|l|l|l|l|l|l|l|l}
\hline & $\mathrm{V} 1 / 2 \mathrm{mar}$ & $\mathrm{V} 3.0$ & $\mathrm{~V} 3.5$ & $\mathrm{~V} 4.0$ & $\mathrm{~V} 4.5$ & $\mathrm{~V} 5.0$ & $\mathrm{~V} 5.5$ & Uma das possíveis interpretações \\
\hline$\underline{\mathrm{x}}$ & 5.36 & 5.20 & 5.28 & 5.33 & 5.39 & 5.43 & 5.48 \\
\hline$\pm \mathrm{dp}$ & 0.25 & 0.23 & 0.22 & 0.22 & 0.22 & 0.22 & 0.22 & com base nos resultados do modelo de \\
\hline
\end{tabular}


mente é possível obter estimativas da performance na meia-maratona partindo, exclusivamente, da concentração de lactato expressa em função de determinada velocidade de corrida. De fato, alguns investigadores consideram ser possível prever a performance competitiva a partir unicamente de um simples teste de terreno $^{3}, 5,13$. Numa investigação semelhante conduzida com o objetivo de predizer o resultado na maratona, os autores concluíram que era possível efetuar essa previsão com um erro aproximado de $\pm 3 \mathrm{~min}$ a partir do cálculo da $V_{2.5}$ num teste de terreno similar ao que utilizamos neste estudo ${ }^{5}$. No entanto, um estudo mais recente $^{17}$ sugere que só as previsões da performance baseadas $\mathrm{naV}_{1.5} \mathrm{enaV}_{2.0}$ apresentam significado fisiológico e asseguram uma boa eficácia em termos de previsão, com um baixo erro padrão de estimativa $(0.141$ e $0.124 \mathrm{~m} / \mathrm{s}$, respectivamente) e assegurando uma margem de erro final entre 4'59" (VI.5) e 5'40" (V2.0).

No entanto, convém ter presente que o comportamento do atleta em situação de competição está dependente de uma enorme quantidade de fatores que, em maior ou menor grau, são susceptíveis de influenciar o seu rendimento. A prestação competitiva não deve ser analisada de uma forma demasiado estrita e confinada a um único indicador, uma vez que se trata de uma questão complexa e multifatorial. Deste modo, apesar dos resultados de ambos os estudos sugerirem a possibilidade de uma previsão da performance a partir das concentrações de lactato sangüíneo, parece-nos, de certa forma, ousado tentar predizer o comportamento tanto na maratona como na meia-maratona, exclusivamente a partir do valor de uma única variável. Com efeito, a ausência de um modelo conceptual que apresente o quadro hierárquico e interativo das variáveis que condicionam a performance na maratona impede que se atribua qualquer generalização tanto em relação ao referido trabalho como à nossa própria investigação.

Um outro fato que importa mais uma vez referir é que encontramos correlações elevadas (com apenas ligeiras diferenças) para todas as hipóteses testadas, o que poderia significar que entre V3.0-V5.5 todas as concentrações poderiam assegurar boas previsões! Se tomarmos como exemplo um dos atletas de elite da amostra que concluiu a sua prova em lh01'51" ( $21095 \mathrm{~m})$ e efetuarmos os cálculos anteriores, verificamos que a previsão para o seu tempo final corresponderá a uma velocidade situada entre $5.47(\mathrm{~V} 3.0)$ e $5.78 \mathrm{~m} / \mathrm{s}$ (V5.5) e por uma marca entre lh04'16"e lh00'50"! Ora uma previsão com um erro de 3' 16" apresenta pouca eficácia preditiva, apresentando por isso pouca aplicabilidade prática. Já situando a previsão entre V4.5-V5.5 diminuímos a margem de erro para 1'23", correspondendo a um tempo final situado entre lh02' 13"-lh00'50". No entanto, para além de em termos práticos ainda ser uma margem de erro elevada, importa referir que $70 \%$ dos atletas visados nesta investigação tenham apresentado um tempo de competição que se situou fora do intervalo de uma previsão baseada na V4.5-V5.5.

A este propósito, uma outra crítica que pode ser feita ao estudo de Fõhrenbach et ai. ${ }^{5} e ́$ que para os mesmos valores de correlação e para equações de regressão semelhantes, os autores concluem, sem fornecer qualquer explicação, que a melhor predição é obtida para valores de 2.5-3.0mmol/1.

Uma outra limitação relativa a estes dois trabalhos é o fato de não haver qualquer pesquisa de validação cruzada para as equações obtidas. Efetivamente, a inexistência de estudos semelhantes realizados com diferentes amostras não permitiu ainda confrontar os nossos resultados com os de outros investigadores. Deste modo, a ausência de validação cruzada pode sugerir que os resultados obtidos emergem de idiossincrasias próprias da amostra.

Os resultados relativos à lactatemia máxima no final da competição estão de acordo com os dados obtidos com base nos testes de terreno. O fato das correlações mais elevadas terem sido obtidas para as intensidades situadas entre V4.5-V5.5 significa que a intensidade a que é corrida a meia-maratona é superior à velocidade correspondente ao limiar das $4 \mathrm{~m}$ $\mathrm{mol} / 1\left(\mathrm{~V}_{4}\right)$. Deste modo, os atletas não correm

\section{A prestação competitiva não deve ser analisada de uma forma demasiado estrita e confinada a um único indicador, uma vez que se trata de uma questão complexa e multifatorial.}


em equilíbrio, mas sim em regime de acumulação progressiva de lactato, o que é bem ilustrado pelo valor médio de $6.47 \pm 1.06 \mathrm{mmol} / 1$ de lactatemia final.

Temos forçosamente de referir um fator que pode, de algum modo, ter tido alguma influência nos resultados finais que obtivemos. Como pode ser observado na figura 2, não existe uma distribuição muito homogênea dos pontos nos vários diagramas referentes às retas de regressão. Isto foi devido à escassez de resultados na competição no intervalo situado entre $\mathrm{hOV}$ $(5.25 \mathrm{~m} / \mathrm{s})$ e lhlO' $^{\prime}(5.02 \mathrm{~m} / \mathrm{s})$. De fato, as marcas obtidas nas várias meias-maratonas pelos atletas que compunham a nossa amostra foram algo heterogêneas, com $67 \%$ dos corredores a efetuarem as suas provas em menos de lh06' e $21 \%$ a registarem resultados acima da $1 \mathrm{hlO}^{\prime}$. De qualquer modo, não nos parece que os resultados encontrados tivessem sido substancialmente diferentes se a distribuição se apresentasse mais homogênea.

Para concluir, gostaríamos de referir que os doseamentos de lactato sangüíneo, apesar de constituírem um indicador extremamente viável na avaliação e prescrição do exercício, devem ser utilizados de forma criteriosa, tendo sempre presente, por um lado, a grande quantidade de informação que podem fornecer, mas igualmente os limites próprios inerentes à sua utilização.

\section{REFERÊNCIAS BIBLIOGRÁFICAS}

1. BILLAT L.V. Use of blood lactate measurements for prediction of exercise performance and for control of training. Recommendations for longdistance running. Sports Med. 22(3): 15775,1996

2. CISAR C.J., THORLAND W.G., JOHNSON G.O. e Housh T.J. The effect of endurance training on metabolic responses and the prediction of distance running performance. J. Sports Med. Phys. Fitness 26(3):234-40,1986

3. FÕHRENBACH, R. Leistungsdiagnostik, Trainingsanalyse und -steuerung bei Lauferinnen und Laufern verschiedener Laufdisziplinen. Hartung-Gorre Verlag, Konstanz, 1991.
4. FOHRENBACH, R., LIESEN, H., MADER, A., HECK, H. e HOLLMANN, W. Die Ausdauerleistungsfähigkeit deutscher Spitzenathletinnen mit Wettkampfstrecken vom Sprint bis zum Marathonlauf. In: Sport: Leistung und Gesundheit, Deutscher SportärztekongreB 1982. Ed. Heck H., Hollmann W., Liesen H. e Rost R. Deutscher Ärzte-Verlag, Kõln, 1983.

5. FOHRENBACH, R., MADER, A. e HOLLMANN, W. Determination of endurance capacity and prediction of exercise intensities for training and competition in marathon runners. Int. J. Sports Med. 8 (1):11-18, 1987

6. FOHRENBACH, R., MADER, A. e HOLLMANN W. Umfang und Intensität im Dauerlauftraining von Mittelstreckenlauferinnen des DLV und MaBnahmen zur individuellen Trainings- und Wettkampfoptimierung. Leistungssport 6:46', 1981.

7. GRANT, S., CRAIG, L, WILSON, J. e AITCHI$\mathrm{SON}, \mathrm{T}$. The relationship between $3 \mathrm{~km}$ running performance and selected physiological variables. J Sports Sci. 15(4):403-10,1997

8. HECK, H. Laktat in der Leistungsdiagnostik. Schorndorf: Hofmann, 1990b.

9. HECK, H. Energiestoffwechsel und medizinische Leistungsdiagnostik. Köln: Schorndorf: Hofmann, 1990a.

10. HECK, H., MADER, A., HESS G, MÜCKE, S., MÜLLER, R. e HOLLMANN, W. Justification of the $4 \mathrm{mmol} / 1$ lactate threshold. Int. J. Sports Med. 6:117-130, 1985

11. KRÜGER, J. Schwimmen und Geländelauf im Modernen Fünfkampf: Sportmedizinische Untersuchungen zur Leistungfähigkeit und entwicklung. Ed. Czwalina Verlag Hamburg, 1995

12. MADER, A. Evaluation of the endurance performance of marathon runners and theoretical analysis of test results. J. Sports Med. Phys. Fitness 31(1):1-19, 1991

13. MADER, A. Evaluation of the endurance performance of marathon runners and theoretical analysis of test results. J. Sports Med. Phys. Fitness 31(1):1-19, 1991.

14. MADER, A. e HECK, H. A theory of the metabolic origin of "anaerobic threshold". Int. J. Sports Med. 1(7):45S-65S, 1986.

15. MADER, A., HECK, H. e HOLLMANN W. Evaluation of lactic acid anaerobic energy contribution oby determination of post-exercise lactic acid concentration of ear capillary blood in middle-distance runners and swimmers. The 
international congress of physical activity Sciences. Quebec, Canada, 1976; Book 4, Exercise physiology. Miami, Florida, 1978.

16. MADSEN, Ö., OLBRECHT, J., MADER, A., LIESEN, H. e HOLLMANN, W. Specifics of aerobic training. In: World Clinic Yearbook. Ed. Ousley R. M. Fort Lauderdale, ASCA, 1983.

17. PAIVA, M. e SANTOS, P.J. Estudo preditivo da intensidade de corrida na maratona a partir da relação lactato-velocidade obtida num teste de terreno. Actas do $1^{\circ}$ Congresso Internacional de Ciências do Desporto, FCDEF-UP, Porto, 1999.

18. ROECKER, K., SCHOTTE, O., NIESS, A.M., HORSTMANN, T.e DICKHUTH, H.H. Predicting competition performance in long-distance running by means of a treadmill test. Med Sci Sports Exerc. 30(10):1552-7,1998.

19. ROGOL, A. Prediction of lactate threshold (LT) and fixed blood lactate concentrations (FBLC) from 3200-m running performance in women. Int J Sports Med. 11(5):373-8,1990.

20. SANTOS, P.J., KRÜGER, J. e HECK, H. Leistungsfähigkeit von Spitzenlangstreckenläufern in Abhängigkeit von Umfang und Intensität des Trainings. In: 9. Internationales Triathlon-Symposium. Ed. Engelhardt M, Franz B., Neumann G. e Pfiitzner A., Hamburg, Czwalina: 257-266, 1995.
21. SCHABORT, E.J., KILLIAN, S.C., GIBSON A.S., HAWLEY, J.A. eNOAKES, T.D. Prediction of triathlon race time from laboratory testing in national triathletes. Med. Sci. Sports Exerc. 32(4):844-9,2000.

22. SLEIVERT, G.G., WENGER, H.A. Physiological predictors of short-course triathlon performance. Med Sci Sports Exerc. 25(7):8716,1993.

23. WELTMAN, A., SNEAD, D., SEIP, R., SCHURRER, R., LEVINE, S., RUTT, R., REILLY, T, WELTMAN, J. e ROGOL, A. Prediction of lactate threshold and fixed blood lactate concentrations from 3200-m running performance in male runners.Int. J. Sports Med. $8(6): 401-6,1987$.

\section{UNITERMOS}

Previsão da performance; limiar aeróbioanaeróbio; lactato; testes de terreno; corredores de elite; meio-fundo.

*Paulo J. M. Santos é Professor auxiliar da Faculdade de Ciências do Desporto e de Educação Física da Universidade do Porto. E-mail: psantos@fcdef.up.pt 